\title{
New molecular targets and lifestyle interventions to delay aging sarcopenia
}

\author{
Fabian Sanchis-Gomar ${ }^{1,2 *}$, Helios Pareja-Galeano ${ }^{1}$, Sara Mayero ${ }^{3}$, Carme Perez-Quilis ${ }^{2}$ and Alejandro Lucia ${ }^{4}$ \\ ${ }^{1}$ Department of Physiology, Faculty of Medicine, University of Valencia and Fundación Investigación Hospital Clínico Universitario INCLIVA, Valencia, Spain \\ ${ }^{2}$ Department of Molecular and Mitochondrial Medicine, University Research Institute "Dr. Viña Giner," Catholic University of Valencia "San Vicente Mártir," Valencia, \\ Spain \\ ${ }^{3}$ Department of Psychiatry, Hospital General Universitario, Valencia, Spain \\ ${ }^{4}$ Universidad Europea and Research Institute of Hospital 12 de Octubre (i+12), Madrid, Spain \\ ${ }^{*}$ Correspondence: fabian.sanchis@uv.es \\ Edited by: \\ Luciano Merlini, Istituto Ortopedico Rizzoli IRCCS, Italy \\ Reviewed by: \\ Enzo Emanuele, Living Research, Italy \\ Nuria Garatachea, University of Zaragoza, Spain
}

Keywords: muscle atrophy, senescence factors, signaling pathways, frailty, pharmaceutical targets

The term sarcopenia was originally created to refer age-related loss of muscle mass with consequent loss of strength (Morley et al., 2001). There are now four international definitions of sarcopenia (CruzJentoft et al., 2010; Muscaritoli et al., 2010; Morley et al., 2011). In essence they all agree, requiring a measure of walking capability [either low gait speed or a limited endurance (distance) in a 6min walk], together with an appendicular lean mass of $<2$ SDs of a sex and ethnically corrected normal level for individuals 20-30 years old. Sarcopenia is a prevalent health problem among the elderly. On average, $5-13$ and $11-50 \%$ of people aged $60-70$ years and $\geq 80$ years, respectively suffer sarcopenia with higher prevalences $(68 \%)$ been reported in nursing home residents $\geq 70$ years (Landi et al., 2012).

Sarcopenia needs to be differentiated from cachexia, which is a combination of both muscle and fat loss and is usually attributable to an excess of catabolic cytokines associated with a disease process (Argiles et al., 2010). Sarcopenia is a prime component of the frailty syndrome, and both sarcopenia and frailty are associated with increased disability, falls, hospitalization, nursing home admission, and mortality (Cesari and Vellas, 2012; Landi et al., 2012).

Medical efforts to develop treatments aiming at preventing aging sarcopenia as well as acute muscle atrophy and frailty in critical patients are considered a step forward in public health. Several hormonal therapies have been proposed for this purpose, such as those based on human growth hormone (hGH), IGF-1, testosterone, and stanozolol. However, the secondary effects associated with these therapies make it necessary to find novel non-toxic and nonhormonal therapies. In this way, elderly or bedridden patients may improve muscle function and decrease the degree of dependence associated with these populations. New drugs such as allopurinol or losartan (Sanchis-Gomar et al., 2011), all of them approved by the Food and Drugs Administration (FDA) and actually prescribed for the treatment of other diseases, could be useful in preventing loss of muscle mass in the described susceptible populations yet new pharmacological targets are needed.

\section{NOVEL PHARMACOLOGICAL TARGETS TO PREVENT SARCOPENIA: EMERGING PATHWAYS TO BE EXPLORED p16INK4a, NAD ${ }^{+}$, AND SESTRINS PATHWAYS}

In a recent manuscript, we proposed new targets for combating aging-related chronic illness (Pareja-Galeano et al., 2014). An altered mitochondrial homeostasis through reduced sirtuin 1 (SIRT1) activity induced by low nicotinamide adenine dinucleotide $\left(\mathrm{NAD}^{+}\right)$levels has been recently advocated as a hallmark of muscle aging. A depleted $\mathrm{NAD}^{+}$pool could be the result of both the diminished $\mathrm{NAD}^{+}$synthesis and increased $\mathrm{NAD}^{+}$consumption that occurs with age (Gomes et al., 2014). Treatment of mice with $\mathrm{NMN}$ (an $\mathrm{NAD}^{+}$precursor) can restore $\mathrm{NAD}^{+}$levels and markers of mitochondrial function that decay with age, reversing muscle mitochondrial senescence (Prolla and Denu, 2014).

Another novel potential biomarker arising from recent animal research is the p16INK4a tumor suppressor. In geriatric mice, satellite cells lose their quiescent state owing to deregulation of p16INK4a, whereas repressing p16INK4a restores muscle regenerative capacity (Sousa-Victor et al., 2014). It is also known that p16INK4a expression increases with age, and its greater expression has been linked to increased attrition (Tsygankov et al., 2009). Recent evidence suggests that p16INK4a mRNA expression in peripheral blood T-lymphocytes is upregulated by gerontogenic behaviors such as tobacco use and physical inactivity, pointing to a critical role in age-related diseases (Song et al., 2010).

Sestrins are a third recently discovered hallmark of aging sarcopenia. Mammalian cells express sestrins (Sesn1, Sesn2, and Sesn3) in response to stress including DNA damage, oxidative stress, and hypoxia. Sestrins can inhibit the activity of the mammalian target of rapamycin complex 1 (mTORC1) through activation of AMP-dependent protein kinase (AMPK) (Lee et al., 2013). Sestrins prevent sarcopenia, insulin resistance, diabetes, and obesity. They also extend life and health span through activation of AMPK, suppression of $\mathrm{mTORC1}$, and stimulation of autophagic signaling (Lee et al., 2013). We also proposed a possible role of the AMPK-modulating functions of sestrins in 


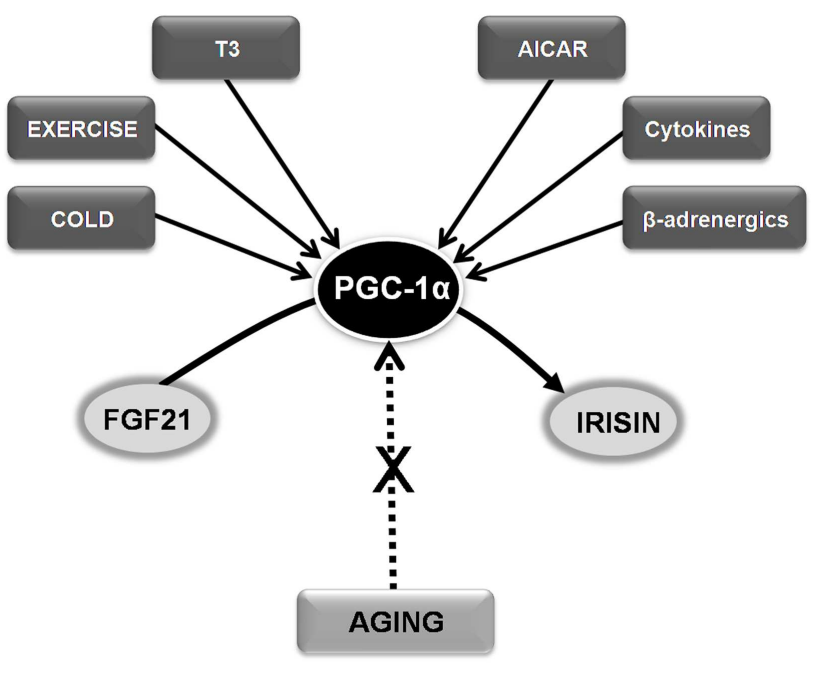

FIGURE 1 | Hypothesizing the role of FGF21-PGC-1 $\alpha$-Irisin axis in age-related conditions and sarcopenia. See text for abbreviations.

the benefits produced by exercise in older subjects (Sanchis-Gomar, 2013a).

\section{FGF21 AND IRISIN: POTENTIAL THERAPEUTIC PGC-1 $\alpha$-RELATED TARGETS FOR AGING AND AGE-ASSOCIATED DISEASES}

Circulating body levels of irisin and fibroblast growth factor 21 (FGF21) increase after cold exposure (Lee et al., 2014). Exercise-induced irisin secretion by working skeletal muscles, which could have evolved from shivering-related muscle contraction, might be a potential target of therapies designed to optimize weight control and metabolic profile (Lee et al., 2014). Hence, targeting irisin and FGF21, and particularly the key signaling molecule responsible for their secretion, the peroxisome proliferator-activated receptor gamma coactivator-1 $\alpha$ (PGC- $1 \alpha$ ), could identify new candidates to be included in the anti-aging armamentarium (SanchisGomar, 2013b).

Irisin is an 112-amino acid glycoprotein, derived from the cleavage in working muscles - and subsequent secretion to the circulation - of a PGC- $1 \alpha$-dependent type I membrane protein, the fibronectin type III domain-containing protein 5 (FNDC5, 209 amino acids) (Bostrom et al., 2012). Exercise-released irisin might act as a hormone either locally within the muscle or targeting distant organs, particularly white adipose tissue, and increase total energy expenditure (Bostrom et al., 2012).
Irisin production increases with chronic endurance exercise in mice and humans, and has been described to mitigate obesity and diet-induced insulin resistance (Bostrom et al., 2012), yet its levels decline with age (Sanchis-Gomar and Perez-Quilis, 2014). To explain exercise benefits on insulin resistance, we recently proposed the following pathway starting in muscle and targeting pancreatic $\beta$-cells: exercise-induced reactive oxygen species $\quad(\mathrm{ROS}) \rightarrow \mathrm{p} 38 \rightarrow$ MAPK $\rightarrow$ PGC$1 \alpha \rightarrow$ irisin $\rightarrow$ betatrophin $\rightarrow \beta$-cell regeneration (Sanchis-Gomar and PerezQuilis, 2014). This pathway could also be affected by aging. Interestingly, it has been also recently reported that disease-free centenarians have increased serum irisin levels (Emanuele et al., 2014). Exercise induces the expression of another PGC- $1 \alpha$ - related hormone, FGF21 (Kim et al., 2013). Fasting drives the production of FGF21 in the liver, where it induces PGC- $1 \alpha$ expression, thereby stimulating fatty acid oxidation, tricarboxylic acid cycle flux, and gluconeogenesis. In effect, mice lacking FGF21 are unable to fully induce PGC-1 $\alpha$ expression in response to a prolonged fast and show impaired gluconeogenesis and ketogenesis (Potthoff et al., 2009). Thus, FGF21 plays an important role in ensuring metabolic regulation during progression from fasting to starvation.

Besides metabolic deregulation and increased insulin resistance, another important consequence of the aging process, reduced mitochondrial biogenesis, is also linked to abnormal PGC- $1 \alpha$ signaling (Sanchis-Gomar and Derbre, 2014). Importantly, an age-related lack of muscle mitochondrial biogenesis can contribute to sarcopenia. PGC- $1 \alpha$ knock-out mice and aged rats show a strikingly similar muscle phenotype: they are unable to express PGC- $1 \alpha$ in response to the stimuli [i.e., exercise training, cold induction, or thyroid hormone (triiodothyronine T3 - treatment)] that naturally up-regulate this molecule in young healthy rats (Derbre et al., 2012). Thus, maintaining normal PGC- $1 \alpha$ responsiveness might help prevent age-related lack of muscle mitochondrial biogenesis (Derbre et al., 2012). In fact, several PGC- $1 \alpha$ activators such as T3, cold induction, 5 -aminoimidazole4-carboxamide-1-beta-D-ribofuranoside (AICAR), $\beta$-adrenergics, cytokines, and exercise have been postulated to prevent aging sarcopenia (Figure 1). The pioneer results by Lee et al. (2014) also suggest that targeting PGC- $1 \alpha$, e.g., using endocrine activators of brown fat function such as irisin and FGF21, might benefit the treatment of other agerelated conditions, particularly metabolic diseases.

\section{FREE-RADICAL THEORY OF AGING QUESTIONED: OTHER MOLECULAR TARGETS TO PREVENT SARCOPENIA ARE NEEDED}

Treatments for age-related and diseaserelated muscle loss might improve active life expectancy in older people, and lead to substantial health-care savings and improved quality of life (Rastogi-Kalyani et al., 2014). However, the results of recent epidemiological studies (Perez et al., 2009) suggest that antioxidant supplementation does not lower the incidence of major ageassociated diseases and might even increase the risk of death in some cases, have questioned the classic free-radical theory of aging (Gladyshev, 2014; Sanchis-Gomar et al., 2014). In fact, evidence mounts that ROS are important mediators of the healthpromoting, life-span-extending capacity of regular exercise, as they play an important signaling role in a multitude of pathways including: angiogenesis, vascular distensibility, and up-regulation of PGC- $1 \alpha$, PGC$1 \alpha /$ nuclear respiratory factor 1-stimulated mitochondrial biogenesis or cytoprotective 
"stress proteins" (heme oxygenase 1, heat shock proteins like HSP60 and HSP70) in muscle (Fiuza-Luces et al., 2013; SanchisGomar and Derbre, 2014). This means that antioxidant interventions are unlikely to help combat sarcopenia. Moreover, antiROS strategies could even aggravate sarcopenia. Thus, a major switch in strategy is proposed and investigators are now focusing on myostatin and follistatin as promising molecular targets of antisarcopenia treatments. Myostatin is a skeletal muscle-specific secreted peptide, pertaining to the transforming growth factor$\beta$ (TGF- $\beta$ ) family member, that inhibits myoblast proliferation and consequently muscle mass/strength by acting as a negative regulator of mTOR-signaling (Garatachea et al., 2013). Mice treated with losartan, an angiotensin II receptor antagonist, were protected against loss of muscle mass and this effect was mediated by activation of the IGF-1/Akt/mTOR pathway (Sanchis-Gomar et al., 2011). These observations highlight the importance of IGF-1/GH balance in longevity and may be of therapeutic interest when targeting the undesirable effects of aging, especially at the muscle level (Sandri et al., 2013).

Myostatin inhibition by agents capable of blocking the myostatin signaling pathway such as ACVR2B (a soluble form of the activin type IIB receptor) could have important applications in the treatment of human muscle degenerative diseases (Lee et al., 2005). In addition, the growth and derived factor (GDF)-associated serum proteins-1 (GASP-1) and 2 (GASP-2), which show competitive binding with proteins capable of inhibiting myostatin, decrease muscle weight and impair muscle regeneration ability in mice (Lee and Lee, 2013). Moreover, the inhibition of the myostatin/activin A signaling pathway is sufficient to induce muscle hypertrophy and can be an effective therapeutic approach for increasing muscle growth in disease settings characterized by satellite cell dysfunction. Finally, the propeptide follistatin, a myostatin antagonist, might be a useful agent for enhancing muscle growth in human therapeutic applications. In fact, increasing follistatin circulating concentrations might help prevent and treat frailty, as well as the cardiometabolic complications associated with androgen-deprivation therapy (Sanchis-Gomar, 2013b).

\section{IMPORTANCE OF LIFESTYLE INTERVENTIONS TO DELAY SARCOPENIA}

Another important tool in the prevention of sarcopenia is physical exercise (some of the molecular pathways involved have been discussed above). Particularly, exercise training programs with resistance (strength) exercises (i.e., movements performed against a specific external force that is regularly increased during training) are especially useful for improving muscle mass or strength in the elderly (Liu and Latham, 2009), including in the oldest-old (people aged 90 years or over) (Fiatarone et al., 1990).

On the other hand, autophagy also plays an important key role both in the modulation of lifespan and sarcopenia (Madeo et al., 2010; Schiavi et al., 2013). Interestingly, autophagy is required to maintain muscle mass and thus to prevent sarcopenia (Masiero et al., 2009; Neel et al., 2013). In effect, failure of autophagy contributes to the sarcopenic phenotype observed in premature aging (Joseph et al., 2013). For this reason, physical exercise and calorie restriction are commonly recommended to prevent sarcopenia since both of them modulate autophagy signaling (Marzetti et al., 2008; Wohlgemuth et al., 2010).

\section{FINAL OPINION}

As an essential step for the prevention of aging-related diseases, and specifically, sarcopenia, more basic research is needed on the main cellular hallmarks of muscle senescence. There is a plethora of potential molecular signals that are candidates to be targeted in future treatment strategies aiming at combating sarcopenia, a devastating effect of aging that is often overlooked.

\section{REFERENCES}

Argiles, J. M., Anker, S. D., Evans, W. J., Morley, J. E., Fearon, K. C., Strasser, F., et al. (2010). Consensus on cachexia definitions. J. Am. Med. Dir. Assoc. 11, 229-230. doi:10.1016/j.jamda.2010.02.004

Bostrom, P., Wu, J., Jedrychowski, M. P., Korde, A., Ye, L., Lo, J. C., et al. (2012). A PGC1-alpha-dependent myokine that drives brown-fat-like development of white fat and thermogenesis. Nature 481, 463-468. doi:10.1038/nature10777

Cesari, M., and Vellas, B. (2012). Sarcopenia: a novel clinical condition or still a matter for research? J. Am. Med. Dir. Assoc. 13, 766-767. doi:10.1016/j. jamda.2012.07.020

Cruz-Jentoft, A. J., Baeyens, J. P., Bauer, J. M., Boirie, Y., Cederholm, T., Landi, F., et al. (2010). Sarcopenia: European consensus on definition and diagnosis: report of the European working group on sarcopenia in older people. Age Ageing 39, 412-423. doi:10.1093/ageing/afq034

Derbre, F., Gomez-Cabrera, M. C., Nascimento, A. L., Sanchis-Gomar, F., Martinez-Bello, V. E., Tresguerres, J. A., et al. (2012). Age associated low mitochondrial biogenesis may be explained by lack of response of PGC-1alpha to exercise training Age (Dordr) 34, 669-679. doi:10.1007/s11357-0119264-y

Emanuele, E., Minoretti, P., Pareja-Galeano, H., Sanchis-Gomar, F., Garatachea, N., and Lucia, A (2014). Serum irisin levels, precocious myocardial infarction, and healthy exceptional longevity. Am. J. Med. doi:10.1016/j.amjmed.2014.04.025

Fiatarone, M. A., Marks, E. C., Ryan, N. D., Meredith, C. N., Lipsitz, L. A., and Evans, W. J. (1990). High-intensity strength training in nonagenarians. Effects on skeletal muscle. JAMA 263, 3029-3034 doi:10.1001/jama.1990.03440220053029

Fiuza-Luces, C., Garatachea, N., Berger, N. A., and Lucia, A. (2013). Exercise is the real polypill. Physiology (Bethesda) 28, 330-358. doi:10.1152/physiol. 00019.2013

Garatachea, N., Pinos, T., Camara, Y., RodriguezRomo, G., Emanuele, E., Ricevuti, G., et al. (2013). Association of the K153R polymorphism in the myostatin gene and extreme longevity. Age (Dordr) 35, 2445-2454. doi:10.1007/s11357-013-9513-3

Gladyshev, V. N. (2014). The free radical theory of aging is dead. Long live the damage theory! Antioxid. Redox Signal. 20, 727-731. doi:10.1089/ ars.2013.5228

Gomes, A. P., Price, N. L., Ling, A. J., Moslehi, J. J., Montgomery, M. K., Rajman, L., et al. (2014). Declining $\mathrm{NAD}(+)$ induces a pseudohypoxic state disrupting nuclear-mitochondrial communication during aging. Cell 155, 1624-1638. doi:10.1016/j. cell.2013.11.037

Joseph, A. M., Adhihetty, P. J., Wawrzyniak, N. R., Wohlgemuth, S. E., Picca, A., Kujoth, G. C., et al. (2013). Dysregulation of mitochondrial quality control processes contribute to sarcopenia in a mouse model of premature aging. PLOS ONE 8:e69327. doi:10.1371/journal.pone.0069327

Kim, K. H., Kim, S. H., Min, Y. K., Yang, H. M., Lee, J. B., and Lee, M. S. (2013). Acute exercise induces FGF21 expression in mice and in healthy humans. PLoS ONE 8:e63517. doi:10.1371/journal. pone.0063517

Landi, F., Liperoti, R., Fusco, D., Mastropaolo, S., Quattrociocchi, D., Proia, A., et al. (2012). Sarcopenia and mortality among older nursing home residents. J. Am. Med. Dir. Assoc. 13, 121-126. doi:10.1016/j. jamda.2011.07.004

Lee, J. H., Budanov, A. V., and Karin, M. (2013). Sestrins orchestrate cellular metabolism to attenuate aging. Cell Metab. 18, 792-801. doi:10.1016/j.cmet. 2013.08.018

Lee, P., Linderman, J. D., Smith, S., Brychta, R. J., Wang, J., Idelson, C., et al. (2014). Irisin and FGF21 are cold-induced endocrine activators of brown fat function in humans. Cell Metab. 19, 302-309. doi:10.1016/j.cmet.2013.12.017

Lee, S. J., Reed, L. A., Davies, M. V., Girgenrath, S., Goad, M. E., Tomkinson, K. N., et al. (2005). Regulation of muscle growth by multiple ligands signaling through activin type II receptors. Proc. Natl. 
Acad. Sci. U.S.A. 102, 18117-18122. doi:10.1073/ pnas. 0505996102

Lee, Y. S., and Lee, S. J. (2013). Regulation of GDF11 and myostatin activity by GASP-1 and GASP2. Proc. Natl. Acad. Sci. U.S.A. 110, E3713-E3722. doi:10.1073/pnas.1309907110

Liu, C. J., and Latham, N. K. (2009). Progressive resistance strength training for improving physical function in older adults. Cochrane Database Syst. Rev. 8:CD002759. doi:10.1002/14651858. CD002759.pub2

Madeo, F., Tavernarakis, N., and Kroemer, G. (2010). Can autophagy promote longevity? Nat. Cell Biol. 12, 842-846. doi:10.1038/ncb0910-842

Marzetti, E., Lawler, J. M., Hiona, A., Manini, T., Seo, A. Y., and Leeuwenburgh, C. (2008). Modulation of age-induced apoptotic signaling and cellular remodeling by exercise and calorie restriction in skeletal muscle. Free Radic. Biol. Med. 44, 160-168. doi:10.1016/j.freeradbiomed.2007.05.028

Masiero, E., Agatea, L., Mammucari, C., Blaauw, B., Loro, E., Komatsu, M., et al. (2009). Autophagy is required to maintain muscle mass. Cell Metab. 10, 507-515. doi:10.1016/j.cmet.2009.10.008

Morley, J. E., Abbatecola, A. M., Argiles, J. M., Baracos, V., Bauer, J., Bhasin, S., et al. (2011). Sarcopenia with limited mobility: an international consensus. J. Am. Med. Dir. Assoc. 12, 403-409. doi:10.1016/j.jamda.2011.04.014

Morley, J. E., Baumgartner, R. N., Roubenoff, R., Mayer, J., and Nair, K. S. (2001). Sarcopenia. J. Lab. Clin. Med. 137, 231-243. doi:10.1067/mlc.2001. 113504

Muscaritoli, M., Anker, S. D., Argiles, J., Aversa, Z., Bauer, J. M., Biolo, G., et al. (2010). Consensus definition of sarcopenia, cachexia and pre-cachexia: joint document elaborated by special interest groups (SIG) "cachexia-anorexia in chronic wasting diseases" and "nutrition in geriatrics". Clin. Nutr. 29, 154-159. doi:10.1016/j.clnu. 2009.12.004

Neel, B. A., Lin, Y., and Pessin, J. E. (2013). Skeletal muscle autophagy: a new metabolic regulator. Trends Endocrinol. Metab. 24, 635-643. doi:10.1016/j.tem. 2013.09.004

Pareja-Galeano, H., Sanchis-Gomar, F., and Lucia, A. (2014). p16INK4a, NAD, and sestrins: new targets for combating aging-related chronic illness? J. Cell. Physiol. doi:10.1002/jcp.24627

Perez, V. I., Bokov, A., Van Remmen, H., Mele, J., Ran, Q., Ikeno, Y., et al. (2009). Is the oxidative stress theory of aging dead? Biochim. Biophys. Acta 1790, 1005-1014. doi:10.1016/j.bbagen.2009.06.003

Potthoff, M. J., Inagaki, T., Satapati, S., Ding, X., He, T., Goetz, R., et al. (2009). FGF21 induces PGC-1alpha and regulates carbohydrate and fatty acid metabolism during the adaptive starvation response. Proc. Natl. Acad. Sci. U.S.A. 106, 10853-10858. doi:10. 1073/pnas.0904187106

Prolla, T. A., and Denu, J. M. (2014). NAD(+) deficiency in age-related mitochondrial dysfunction. Cell Metab. 19, 178-180. doi:10.1016/j.cmet.2014. 01.005

Rastogi-Kalyani, R., Corriere, M., and Ferrucci, L. (2014). Age-related and disease-related muscle loss: the effect of diabetes, obesity, and other diseases. Lancet Diabetes Endocrinol. doi:10.1016/ S2213-8587(14)70034-8

Sanchis-Gomar, F. (2013a). Sestrins: novel antioxidant and AMPK-modulating functions regulated by exercise? J. Cell. Physiol. 228, 1647-1650. doi:10. $1002 /$ jcp. 24338

Sanchis-Gomar, F. (2013b). The skeletal musclemetabolism axis in prostate-cancer therapy. N. Engl. J. Med. 367, 2257-2258. doi:10.1056/ NEJMc1211955

Sanchis-Gomar, F., and Derbre, F. (2014). Mitochondrial fission and fusion in human diseases. N. Engl. J. Med. 370, 1073-1074. doi:10.1056/ NEJMc1316254

Sanchis-Gomar, F., Gomez-Cabrera, M. C., and Vina, J. (2011). The loss of muscle mass and sarcopenia: non hormonal intervention. Exp. Gerontol. 46 , 967-969. doi:10.1016/j.exger.2011.08.012

Sanchis-Gomar, F., Pareja-Galeano, H., and Lucia, A. (2014). The hMTH1 paradox: antioxidants recommended in cancer? DNA Repair (Amst.). doi:10. 1016/j.dnarep.2014.04.010

Sanchis-Gomar, F., and Perez-Quilis, C. (2014). The p38-PGC-1alpha-irisin-betatrophin axis: exploring new pathways in insulin resistance. Adipocyte 3, 67-68. doi:10.4161/adip. 27370

Sandri, M., Barberi, L., Bijlsma, A. Y., Blaauw, B., Dyar, K. A., Milan, G., et al. (2013). Signalling pathways regulating muscle mass in ageing skeletal muscle: the role of the IGF1-Akt-mTOR-FoxO pathway. Biogerontology 14, 303-323. doi:10.1007/s10522013-9432-9

Schiavi, A., Torgovnick, A., Kell, A., Megalou, E., Castelein, N., Guccini, I., et al. (2013). Autophagy induction extends lifespan and reduces lipid content in response to frataxin silencing in C. elegans.
Exp. Gerontol. 48, 191-201. doi:10.1016/j.exger. 2012.12.002

Song, Z., Von Figura, G., Liu, Y., Kraus, J. M., Torrice, C., Dillon, P., et al. (2010). Lifestyle impacts on the aging-associated expression of biomarkers of DNA damage and telomere dysfunction in human blood. Aging Cell 9, 607-615. doi:10.1111/j.14749726.2010.00583.x

Sousa-Victor, P., Gutarra, S., Garcia-Prat, L. Rodriguez-Ubreva, J., Ortet, L., Ruiz-Bonilla, V., et al. (2014). Geriatric muscle stem cells switch reversible quiescence into senescence. Nature 506, 316-321. doi:10.1038/nature13013

Tsygankov, D., Liu, Y., Sanoff, H. K., Sharpless, N. E., and Elston, T. C. (2009). A quantitative model for age-dependent expression of the pl6INK4a tumor suppressor. Proc. Natl. Acad. Sci. U.S.A. 106, 16562-16567. doi:10.1073/pnas.0904405106

Wohlgemuth, S. E., Seo, A. Y., Marzetti, E., Lees, H. A., and Leeuwenburgh, C. (2010). Skeletal muscle autophagy and apoptosis during aging: effects of calorie restriction and life-long exercise. Exp. Gerontol. 45, 138-148. doi:10.1016/j.exger. 2009.11.002

Conflict of Interest Statement: The authors declare that the research was conducted in the absence of any commercial or financial relationships that could be construed as a potential conflict of interest.

Received:09 May 2014; paperpending published: 14 June 2014; accepted: 18 June 2014; published online: 02 July 2014.

Citation: Sanchis-Gomar F, Pareja-Galeano H, Mayero S, Perez-Quilis C and Lucia A (2014) New molecular targets and lifestyle interventions to delay aging sarcopenia. Front. Aging Neurosci. 6:156. doi: 10.3389/fnagi.2014.00156

This article was submitted to the journal Frontiers in Aging Neuroscience.

Copyright (c) 2014 Sanchis-Gomar, Pareja-Galeano, Mayero, Perez-Quilis and Lucia. This is an open-access article distributed under the terms of the Creative Commons Attribution License (CC BY). The use, distribution or reproduction in other forums is permitted, provided the original author(s) or licensor are credited and that the original publication in this journal is cited, in accordance with accepted academic practice. No use, distribution or reproduction is permitted which does not comply with these terms. 